\title{
Infantile Bladder Rupture during Voiding Cystourethrography
}

\author{
Abdol M. Kajbafzadeh, Parisa Saeedi, Ali R. Sina, Seyedmehdi Payabvash, Amirali H. Salmasi \\ Pediatric Urology Research Center, Department of Urology, Children's Hospital Medical Center, \\ Tehran University of Medical Sciences, Tehran, Iran
}

\begin{abstract}
Bladder rupture is rare during infancy and most of reported cases had urethral obstruction or neurogenic bladder. We report two cases of infantile bladder rupture during voiding cystourethrography (VCUG). This report reinforces the criteria for proper VCUG imaging procedure. Consideration of expected bladder volume for body weight, and close monitoring of bladder pressure and injection speed could prevent such complications.
\end{abstract}

Key words: bladder; children; diagnostic imaging; rupture; iatrogenic

Int Braz J Urol. 2007; 33: 532-5

\section{INTRODUCTION}

Voiding cystourethrography (VCUG) is widely applied for the radiological evaluation of the bladder and urethra in children. Bladder rupture during VCUG is exceedingly rare (1). We present two infants with iatrogenic bladder rupture during VCUG performed by radiology staffs in two district hospitals. These infants were referred to our center for further management.

\section{CASE REPORTS}

Case \#1 - A 10-day-old boy, weighing $3.2 \mathrm{~kg}$, was referred to nephrologists with history of prenatal hydronephrosis. On day 7 after birth, ultrasonographic exam confirmed bilateral hydroureteronephrosis, which was severe on the left side and mild on the right side. VCUG was requested to evaluate a possible vesicoureteral reflux (VUR). A $6 \mathrm{~F}$ feeding tube was inserted into the urethra and contrast media was injected using a 50-mL syringe, under fluoroscopic guide. During the first filling cycle, severe left side VUR appeared following injection of $15 \mathrm{~mL}$ of contrast media (Figure-1) and the right VUR appeared in volume of $35 \mathrm{~mL}$. However, the radiographer continued the instillation until the intraperitoneal bladder rupture occurred in volume of $60 \mathrm{~mL}$ (Figure-1). The baby was referred to urologist and immediately underwent abdominal exploration through a Pfannenstiel incision. The bladder dome was the site of a $2 \mathrm{~cm}$ long rupture. The peritoneal cavity was washed with saline and the bladder was closed in two layers using 4-0 polyglactin suture. A Malecot catheter was inserted in the bladder as suprapubic tube. A minivacuum closed drain was left in the perivesical space. The post-operative course was complicated by 


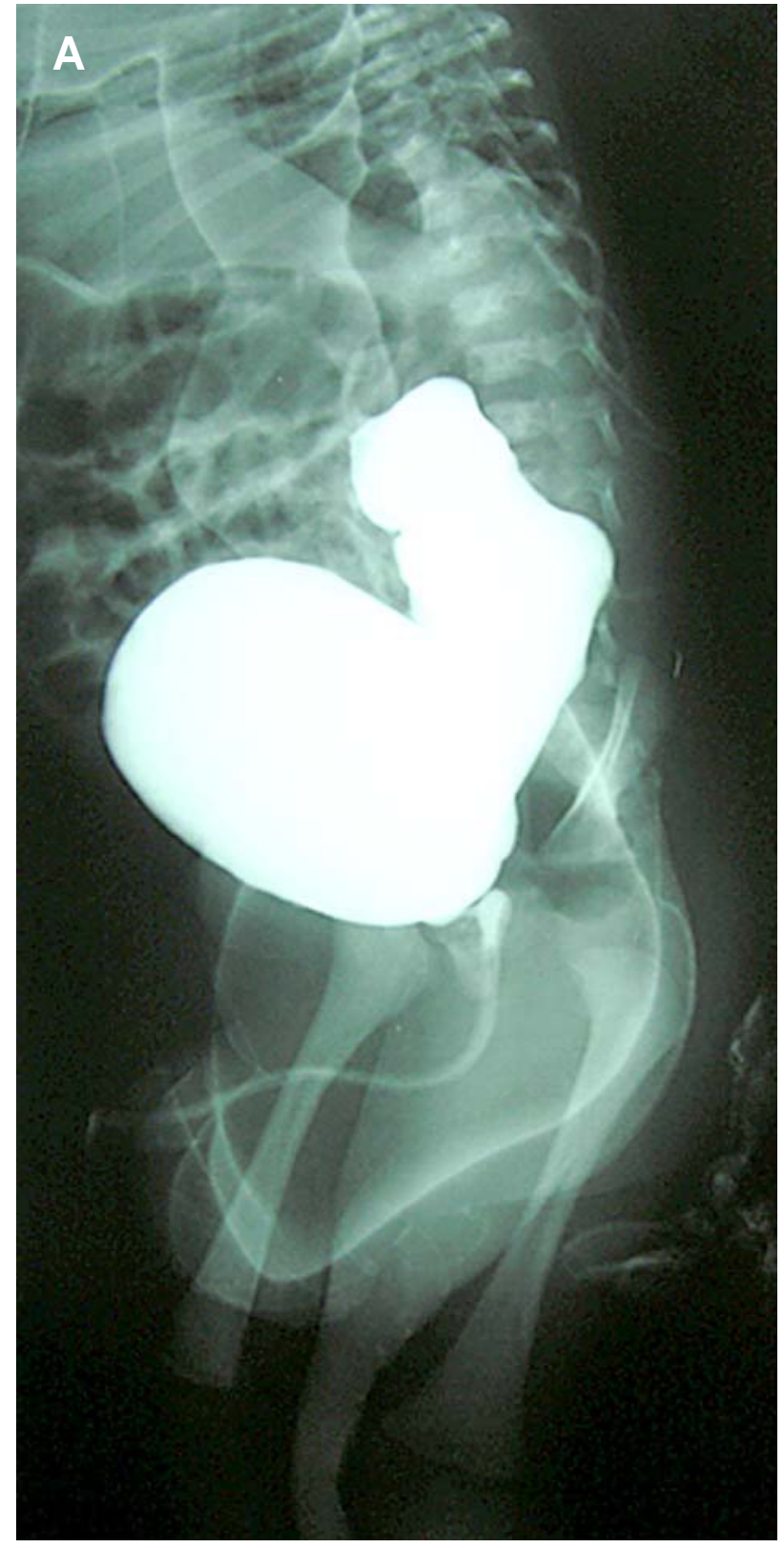

prolonged urine leakage from the site of suprapubic catheter extracted on the $14^{\text {th }}$ postoperative day. The child was referred to our institution for further management. A 6F Foley urethral catheter was inserted. After 7 days, the leakage was stopped and the catheter was removed on the $24^{\text {th }}$ postoperative day. The patient was discharged 3 days later with good condition and prescription of prophylactic antibiotic (Figure-1).

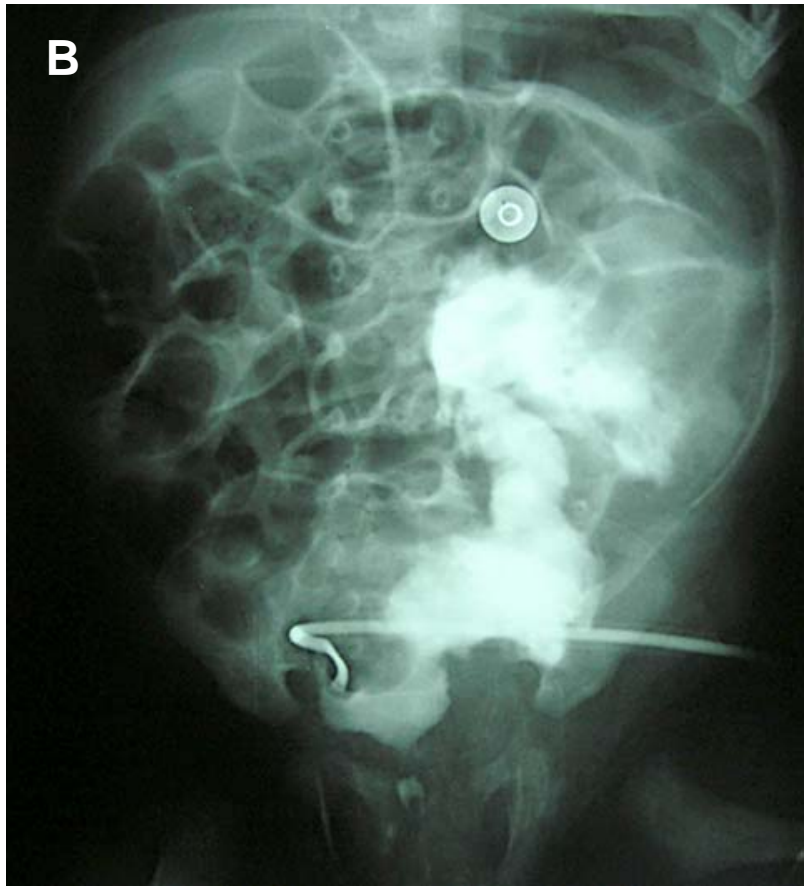

Figure 1 - Voiding cystourethrography of case \#1. A) Highgrade vesicoureteral reflux on the left side. B) Intraperitoneal bladder rupture.

Case \#2 - A 9-month old female infant, weighing $7 \mathrm{~kg}$, was referred to a radiologist for VCUG at a district hospital from a different province. Medical problems included urinary tract infection and failure to thrive. Contrast media was injected through an $8 \mathrm{~F}$ urethral feeding tube under fluoroscopic guide. The radiographer instilled $100 \mathrm{~mL}$ of contrast media using a 50-mL syringe, during the first filling cycle. Speed of injection, bladder pressure and volume were not recorded. While reviewing the images, the radiologist discovered bladder perforation. She was taken to the operating room and underwent abdominal exploration. The bladder was exploded at dome with a $3 \mathrm{~cm}$ length. The site of perforation was closed in two layers using absorbable 4-0 polygalactin suture. A small Penrose drain was left in perivesical space and a $10 \mathrm{~F}$ catheter was left per urethra. Post-operative course of the patient was uneventful and she was discharged one week later and referred to our clinic for further evaluation. Follow-up VCUG showed no leakage or reflux. 

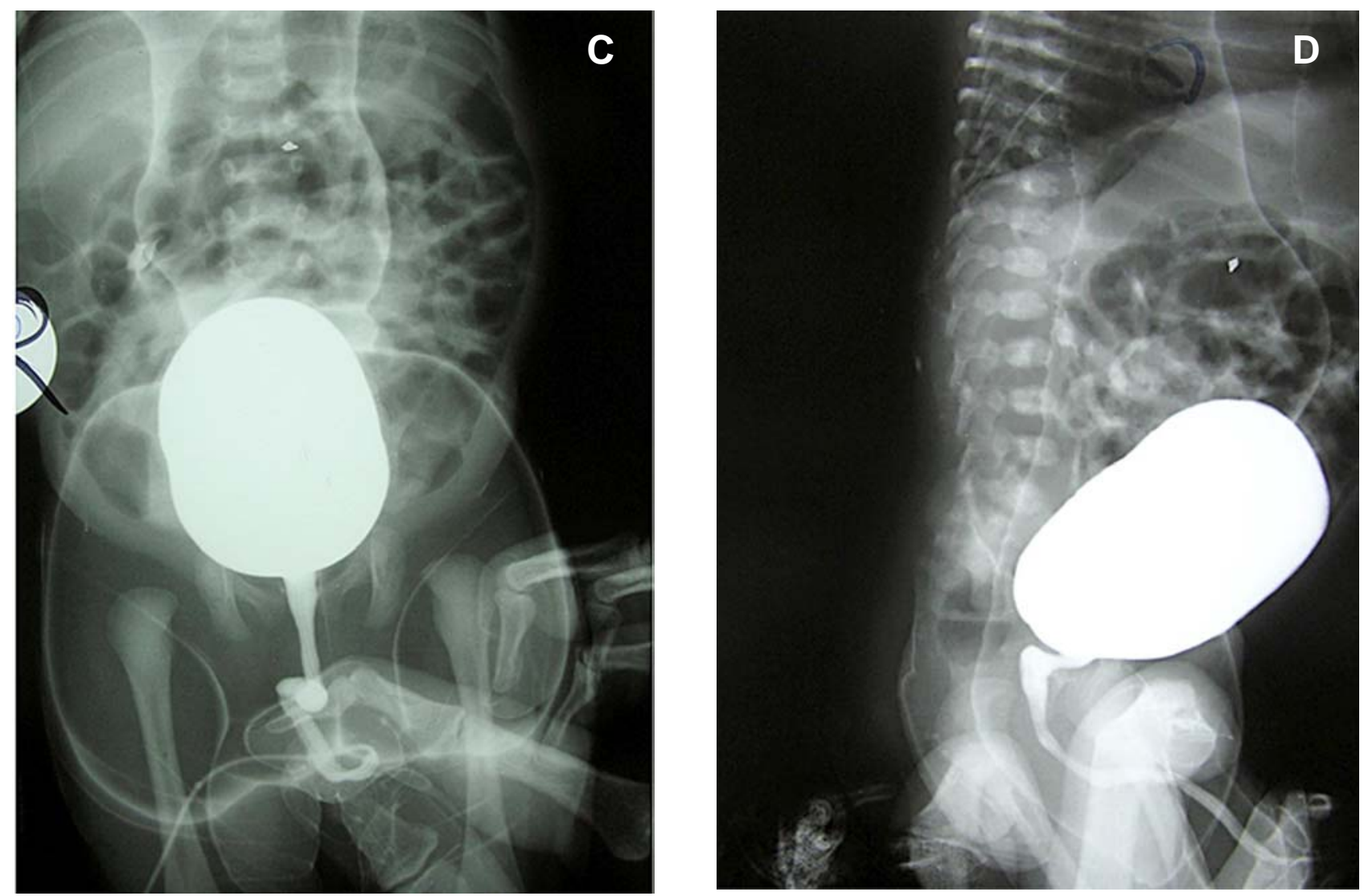

Figure 1 - Voiding cystourethrography of case \#1.C) and D) Frontal and lateral voiding cystourethrography views of the same patient after surgical repair.

\section{COMMENTS}

Infantile bladder rupture is rare (2) and only 17 cases have been reported between 1956 and 1985 (3). The main predisposing factors include posterior urethral valves and neurogenic bladder followed by bladder outlet obstruction from other etiologies and trauma (3). Few cases of iatrogenic bladder perforation have been reported in children following diagnostic and therapeutic procedures $(3,4)$. To our knowledge this is the second report of infantile bladder rupture during VCUG (2).

There was none of the above-mentioned risk factors in our cases; however, inaccurate imaging procedure seems to be the main cause of perforation. In order to perform a safe and perfect VCUG, radiologists must pay attention to some factors such as bladder volume, style of contrast media instillation and patient conditions (underlying urinary disease) (1).
Two formulae have been proposed for bladder volume estimation in children with regards to their weight and age $(4,5)$; age $<2$ years - bladder volume $(\mathrm{mL})=$ weight $(\mathrm{kg}) \times 7$, age $>2$ years - bladder volume $(\mathrm{mL})$ $=[$ age $($ years $)+2] \times 30$.

Proper catheter insertion, fluoroscopic guide, pressure and number of filling cycle should be considered in styles of instillation (1). To avoid pressure overload, hand injection of contrast material must not be used and the contrast container should not be placed higher than $60 \mathrm{~cm}$ from the patient. More than two cycles of filling does not appear to be necessary $(1,6)$. The underlying urinary system disease is another important factor. In our cases, the bladder volume and pressure were not considered and the contrast media was instilled directly by syringe.

Management of infantile bladder rupture should be individualized. In the review by Trulock et al. (3), the majority of reported neonates were treated 
with abdominal exploration and repair of the bladder leakage site; however some of the patients would be managed by the use of vesicostomy or urethral catheter alone.

In conclusion, during VCUG, it is important to consider the patient underlying disorder and expected bladder volume for age as well as to avoid hand injection of contrast material and placing the contrast container more than $60 \mathrm{~cm}$ higher than the patient. Moreover, in order to prevent high-pressure voiding in premature infants, it has been recommended to use small caliber and balloon-less feeding catheters that would not occlude the bladder neck during voiding.

\section{CONFLICT OF INTEREST}

None declared.

\section{Correspondence address:}

Dr. A.M. Kajbafzadeh

No. 36, 2nd Floor, 7th Street

Saadat-Abad, Ave. Tehran 19987, Iran

Fax: + 9821 2206-9451

E-mail:kajbafzd@sina.tums.ac.ir

\section{EDITORIAL COMMENT}

The authors of this manuscript present two cases of iatrogenic bladder rupture in infants undergoing voiding cystourethrograms. This radiographic study is one of the most common imaging studies ordered in children and needs to be performed safely and reliably. Those of us who work in dedicated children's hospitals take this for granted. However, the cases reported in this series were performed by radiologists clearly unfamiliar with proper technique as nicely outlined by the authors. It is important to remember that the peritoneum drapes quite anteriorly

\section{REFERENCES}

1. Agrawalla S, Pearce R, Goodman TR: How to perform the perfect voiding cystourethrogram. Pediatr Radiol. 2004; 34: 114-9.

2. Wosnitzer M, Shusterman D, Barone JG: Bladder rupture in premature infant during voiding cystourethrography. Urology. 2005; 66: 432.

3. Trulock TS, Finnerty DP, Woodard JR: Neonatal bladder rupture: case report and review of literature. J Urol. 1985; 133:271-3.

4. O'Brien WJ, Ryckman FC: Catheter-induced urinary bladder rupture presenting with pneumoperitoneum. J Pediatr Surg. 1994; 29: 1397-8.

5. Koff SA: Estimating bladder capacity in children. Urology. 1983; 21: 248-52.

6. Jequier S, Jequier JC: Reliability of voiding cystourethrography to detect reflux. AJR Am J Roentgenol. 1989; 153: 807-10.

Accepted after revision: April 27, 2007

in small children thus making a rupture very likely to be intraperitoneal, as in these two cases, and therefore surgical exploration is required.

Dr. Lane S. Palmer Chief, Pediatric Urology Schneider Children's Hospital North Shore-Long Island Jewish Health System New York, NY, USA E-mail:lpalmer@nshs.edu 